\title{
The Prouhet Tarry Escott Problem: A Review
}

\author{
Srikanth Raghavendran ${ }^{1,+}$ and Veena Narayanan ${ }^{2, *,+}$ \\ 1 TATA Realty-SASTRA Srinivasa Ramanujan Research Chair for Number Theory, SASTRA Deemed \\ University, Thanjavur 613401, India; srikanth@maths.sastra.edu \\ 2 Department of Mathematics, SASTRA Deemed University, Thanjavur 613401, India \\ * Correspondence: veenanarayanan@sastra.ac.in \\ + These authors contributed equally to this work.
}

Received: 28 December 2018; Accepted: 24 February 2019; Published: 1 March 2019

\begin{abstract}
This paper presents a review of the Prouhet Tarry Escott problem. The solutions of the Prouhet Tarry Escott problem are significant because of its numerous applications. Available literature about the present topic has been critically examined. The ideal and non-ideal symmetric solutions of the problem are pointed out. The present work also aims to familiarize one with the different existing methods of obtaining the solutions of the Tarry Escott problem. Difficulties and possible future research directions are addressed. This review contributes a clear picture of the Prouhet Tarry Escott problem.
\end{abstract}

Keywords: Diophantine systems; equal sums of like powers; Prouhet Tarry Escott problem

\section{Introduction}

Number theory is one of the major streams of pure mathematics, which deals with the study of the positive integers and is extensively found in several aspects of nature. Even though there are a wide range of well-known results, number theory flourishes with many unsolved problems. One of the exciting topics in number theory is the study on Diophantine equations, which mainly emphasizes the problem of finding rational (integral) solutions of a polynomial with integer coefficients. Diophantine problems have been studied by various mathematicians worldwide for so many years. Recently, Srikanth and Subburam [1-3] studied Diophantine equations and contributed vastly to this area.

The problem of equal sums of like powers is to obtain two distinct sets of integers such that the sum of the $k$ th powers of the integers in both the sets is equal for different values of $k$. That is, the problem aims to obtain non-trivial solutions of the Diophantine system:

$$
\sum_{i=1}^{s} x_{i}^{k}=\sum_{i=1}^{s} y_{i}^{k} ; k=k_{1}, k_{2}, \cdots, k_{n},
$$

where the $k_{i}{ }^{\prime} s, x_{i}{ }^{\prime}$ s, and $y_{i}{ }^{\prime}$ s are all integers. If we consider these $k_{i}{ }^{\prime} s$ as consecutive integers then this definition reduces to the definition of the classical Prouhet Tarry Escott (PTE) problem. So the PTE problem falls under the more general problem of equal sums of like powers.

The traditional way of solving the PTE problem is by considering the solutions over the ring of integers, $\mathbb{Z}$. But some studies have also taken place over arbitrary rings, especially on some unique factorization domains. Even though most of the available solutions of the PTE problem for small sizes were found manually, some algorithmic studies were also carried out in recent years to obtain the solutions of PTE problems with size greater than or equal to 12. Almost all of the available work on the problem is presented in Section 3 and is explained in chronological order. 


\section{Preliminaries}

In this section, the authors recall some basic definitions and results that play a significant role in the study of the PTE problem.

Definition 1. The Prouhet Tarry Escott problem aims to obtain two distinct sets of integers say $\mathbb{U}=$ $\left\{u_{1}, u_{2}, \cdots u_{n}\right\}$ and $\mathbb{V}=\left\{v_{1}, v_{2}, \cdots v_{n}\right\}$ such that:

$$
\begin{aligned}
u_{1}+u_{2}+\cdots+u_{n} & =v_{1}+v_{2}+\cdots+v_{n} \\
u_{1}^{2}+u_{2}^{2}+\cdots+u_{n}^{2} & =v_{1}^{2}+v_{2}^{2}+\cdots+v_{n}^{2} \\
\vdots & =\vdots \\
u_{1}^{k}+u_{2}^{k}+\cdots+u_{n}^{k} & =v_{1}^{k}+v_{2}^{k}+\cdots+v_{n}^{k}
\end{aligned}
$$

where $n$ is known as the size and $k$ is known as the degree. The solution sets $\mathbb{U}$ and $\mathbb{V}$ are usually represented as $\mathbb{U}={ }_{k} \mathbb{V}$.

Example 1. The two sets $U=\{26,4,40,8,13,35,-1,31\}$ and $V=\{8,16,10,44,31,23,29,-5\}$ satisfy the conditions:

$$
\begin{aligned}
26+4+40+8+13+35+-1+31 & =8+16+10+44+31+23+29+-5 \\
26^{2}+4^{2}+40^{2}+8^{2}+13^{2}+35^{2}+(-1)^{2}+31^{2} & =8^{2}+16^{2}+10^{2}+44^{2}+31^{2}+23^{2}+29^{2}+(-5)^{2} \\
26^{3}+4^{3}+40^{3}+8^{3}+13^{3}+35^{3}+(-1)^{3}+31^{3} & =8^{3}+16^{3}+10^{3}+44^{3}+31^{3}+23^{3}+29^{3}+(-5)^{3} .
\end{aligned}
$$

Thus, these two sets serve as a solution of Prouhet Tarry Escott problem of degree 3 and size 8 .

If the size and the degree of the PTE problem differ by one (i.e., degree = size -1 ), then the solutions are known as ideal solutions, otherwise they are called non-ideal solutions. For example, the two distinct sets of integers $\{3,7\}$ and $\{4,6\}$ constitute an ideal solution of the PTE problem of size 2 and degree 1 whereas the sets $\{-29,88,-5,46\}$ and $\{13,70,61,-44\}$ exemplify a non-ideal solution of the PTE problem of degree 2 and size 4 .

Note 1. A solution of the PTE problem in which $u_{i}^{\prime}$ s merely form a permutation of $v_{i}^{\prime} s$ is called trivial.

Definition 2. Consider the PTE problem of odd size $n$. Suppose the set of integers, say $\left\{p_{1}, p_{2}, \cdots p_{n}\right\}$ satisfies the system of equations $\sum_{i=1}^{n} p_{i}^{k}=0 ; k=1,3, \cdots, n-1$, then $\left\{p_{1}, p_{2}, \cdots p_{n}\right\}={ }_{n-1}\left\{-p_{1},-p_{2}, \cdots-\right.$ $\left.p_{n}\right\}$. In this case, the solution is called an odd ideal symmetric solution. If the size of the PTE problem is even and suppose $\sum_{i=1}^{n / 2} p_{i}^{k}=\sum_{i=1}^{n / 2} q_{i}^{k} ; k=2,4, \cdots, n-2$, where the $q_{i}^{\prime}$ s are positive integers, then:

$$
\left\{p_{1}, p_{2}, \cdots p_{n / 2}, \cdots,-p_{1},-p_{2}, \cdots-p_{n / 2}\right\}={ }_{n-1}\left\{q_{1}, q_{2}, \cdots q_{n / 2}, \cdots,-q_{1},-q_{2}, \cdots-q_{n / 2}\right\},
$$

and in this case the solution becomes an even ideal symmetric solution.

Example 2. The sets $\{0,2\}$ and $\{1,1\}$ provide an ideal symmetric solution, whereas $\{1,8,8\}$ and $\{2,5,10\}$ give an ideal non-symmetric solution for the PTE problem of $k=1$ and $k=1,2$, respectively.

One can also define the constant associated with the PTE problem as follows: 
Definition 3. Suppose that $\mathbb{U}=\left\{u_{1}, u_{2}, \cdots u_{n}\right\}$ and $\mathbb{V}=\left\{v_{1}, v_{2}, \cdots v_{n}\right\}$ are two sets of positive integers satisfying $\mathbb{U}={ }_{k} \mathbb{V}$ with $k \leq n-1$. Then statements (1) and (2) are equivalent:

$$
\begin{array}{r}
\sum_{i=1}^{n} u_{i}^{l}=\sum_{i=1}^{n} v_{i}^{l} ; \quad l=1,2, \cdots, k . \\
\operatorname{deg}\left(\prod_{i=1}^{n}\left(z-u_{i}\right)-\prod_{i=1}^{n}\left(z-v_{i}\right)\right) \leq n-k-1
\end{array}
$$

This implies that $\mathbb{U}={ }_{n-1} \mathbb{V}$ and $\prod_{i=1}^{n}\left(z-u_{i}\right)-\prod_{i=1}^{n}\left(z-v_{i}\right)=K_{n}$ is a constant. This constant is known as the constant associated with the PTE problem.

It is to be noted that the equations in Definition 3 are derived from the concept of polynomial equivalence (Newton's equations).

\section{General Theorems}

One of the fundamental results on solutions of the PTE problem is due to Frolov [4].

Theorem 1. If $\left\{x_{1}, x_{2}, \cdots, x_{n}\right\}={ }_{k}\left\{y_{1}, y_{2}, \cdots, y_{n}\right\}$, then $\left\{M x_{1}+K, \cdots, M x_{n}+K\right\}={ }_{k}\left\{M y_{1}+\right.$ $\left.K, \cdots, M y_{n}+K\right\}$ where $M$ and $K$ are arbitrary integers.

If one generates a new solution from an existing solution by the repeated application of Theorem 1 , then these two sets of solutions are called equivalent solutions. Solutions which are not equivalent are considered while discussing distinct solutions. In 1910, Escott [5] established Theorem 2.

Theorem 2. If $F(x)$ and $G(x)$ are two polynomials with integral coefficients having their first $r$ terms alike, then $F(x) G(x+d)$ and $G(x) F(x+d)$ where $d$ is an integer, are two polynomials having their first $r+1$ terms alike.

Later Tarry [6] developed a theorem similar to Theorem 2.

Theorem 3. If $\left\{x_{1}, \cdots, x_{n} ; y_{1}, \cdots, y_{n}\right\}$ is a solution set of the PTE problem of degree $k$, then $\left\{x_{1}, \cdots, x_{n}, y_{1}+\right.$ $\left.h, \cdots, y_{n}+h ; x_{1}+h, \cdots, x_{n}+h, y_{1}, y_{2}, \cdots, y_{n}\right\}$ where $h$ is an arbitrary integer, is a solution of the PTE problem of degree $k+1$.

Another important result on the PTE problem is due to Bastien [7] which is stated as follows.

Theorem 4. If the system of equations $\sum_{i=1}^{m} u_{i}^{k}=\sum_{i=1}^{m} v_{i}^{k} ; k=1,2, \cdots, n$, has a non-trivial solution, then $m \geq k+1$.

Theorem 5 ([8]). If $\left\{a_{1}, \cdots, a_{m}\right\}={ }_{k}\left\{b_{1}, \cdots, b_{m}\right\} ; k=1,3, \cdots, 2 n-1$, then $\left\{T+a_{1}, \cdots, T+\right.$ $\left.a_{m}, T-b_{1}, \cdots, T-b_{m}\right\}={ }_{k}\left\{T+b_{1}, \cdots, T+b_{m}, T-a_{1}, \cdots, T-a_{m}\right\} ; k=1,2, \cdots, 2 n$ where $T$ is an arbitrary integer.

Theorem 6. Let $\left\{x_{1}, \cdots, x_{n}\right\}={ }_{n-1}\left\{y_{1}, \cdots, y_{n}\right\}$ be two multisets of integers that constitute an ideal PTE solution, and suppose that a prime $p$ divides the constant $K_{n}$ associated with this solution. Then we can reorder the integers $y_{i}$ so that $x_{i} \equiv y_{i}(\bmod p)$ for $i=1, \cdots, n$.

Let $n \geq 2$ and suppose $a_{i}, 1 \leq i \leq n$, satisfy $0 \leq a_{i} \leq s-1$. Any set $\left\{a_{1}, \cdots, a_{n}\right\}$ such that $a_{1}+a_{2}+\cdots+a_{n} \equiv r(\bmod s)$ is denoted as an $(n, r)$ set. If $r \equiv t(\bmod s)$, every $(n, r)$ set is an $(n, t)$ set and conversely. If $\phi=\phi\left(a_{1}, \cdots, a_{n}\right)$, we say that $\sum_{(n, r)} \phi$, the sum of $\phi$ over all $(n, r)$ sets and is 
independent of $r$ if $\sum_{(n, 0)} \phi=\sum_{(n, 1)} \phi=\cdots \sum_{(n, s-1)} \phi$.

Now, let us recall the well known Lehmer's result on equal sums of like powers.

Theorem 7. If $\mu_{1}, \cdots, \mu_{n}$ are any numbers and $\xi=a_{1} \mu_{1}+a_{2} \mu_{2}+\cdots+a_{n} \mu_{n}$, then $\sum_{(n, r)} \xi^{h}$ is independent of $r$ for $1 \leq h \leq n-1$.

Another interesting idea used in the study of PTE problems is the concept of Prouhet-Thue-Morse sequence.

Definition 4. The Prouhet-Thue-Morse sequence is a binary sequence starting with 0 and the nth element is defined as $t_{n}=1$ if the number of ones in the binary expansion of $n$ is odd, and $t_{n}=0$ if the number of ones in the binary expansion of $n$ is even.

Theorem 8, originally given by Prouhet, illustrates the relation between the Prouhet-Thue-Morse sequence and the PTE problem.

Theorem 8 ([9]). The Prouhet-Thue-Morse sequence $t=\left(t_{n}\right)_{n>0}$ has the following property. Define $I=\{i \in$ $\left.\left\{0,1,2,3, \cdots, 2^{N}-1\right\}: t_{i}=0\right\}, J=\left\{j \in\left\{0,1,2,3, \cdots, 2^{N}-1\right\}: t_{j}=1\right\}$. Then for $0 \leq k \leq N-1$, we have $\sum_{i \in I} i^{k}=\sum_{j \in I} j^{k}$.

Remark 1. Two important facts to be considered in the study of the PTE problem are as follows:

1. No integer can appear more than twice among either $\mathbb{U}$ or $\mathbb{V}$.

2. It is not possible to have three consecutive integers on one side of the solution or two repeated integers and a consecutive integer.

\section{On the Prouhet Tarry Escott Problem}

There have been a lot of studies performed on the solutions of the PTE problem worldwide. It was Euler [10] who made the first contribution to the PTE problem. He remarked that $\left\{t_{1}, t_{2}, t_{3}, t_{1}+t_{2}+t_{3}\right\}={ }_{2}\left\{t_{1}+t_{2}, t_{2}+t_{3}, t_{1}+t_{3}\right\}$ where $t_{1}, t_{2}, t_{3}$ are positive integers. Later, Goldbach [11] pointed out in a letter to Euler that if $p, q, r$, and $s$ are positive integers, then:

$$
\{p+q+s, p+r+s, q+r+s, s\}=2\{p+s, q+s, r+s, p+q+r+s\},
$$

and the findings of Euler was a special case, $s=0$, of what Goldbach noticed. Pollock [12] and Proth [12] also provided some solutions to this problem as a continuation of Goldbach's findings. Cesaro [12] demonstrated that the integers $1,2, \cdots, 9$ satisfies the relation:

$$
\{2,4,9,5\}={ }_{2}\{5,1,6,8\}={ }_{2}\{8,3,7,2\},
$$

and showed some trigonometrical properties of these three sets of four integers.

Later, Prouhet [12] noted that the first 27 integers; $1,2, \cdots, 27$ could be separated into three sets out of which two sets say $X=\{1,6,8,12,14,16,20,22,27\}$ and $Y=\{2,4,9,10,15,17,21,23,25\}$ satisfy $1+6+8+12+14+16+20+22+27=2+4+9+10+15+17+21+23+25$ as well as $1^{2}+6^{2}+8^{2}+12^{2}+14^{2}+16^{2}+20^{2}+22^{2}+27^{2}=2^{2}+4^{2}+9^{2}+10^{2}+15^{2}+17^{2}+21^{2}+23^{2}+25^{2}$. Prouhet generalized this property of integers as: "The $n^{m}$ positive integers can be separated into $n$ sets consisting of $n^{m-1}$ terms so that the sum of the $k$ th powers; $(k<m)$ of terms is the same for all these sets". Even though Prouhet contributed a first solution to the PTE problem, the problem was known as Tarry Escott problem or equal size power's problem until the 20th century when Prouhet's solution was rediscovered. Now the problem is generally termed as the Prouhet Tarry Escott problem. 
Similar kinds of earlier studies on the PTE problem can be found in Reference [12], which contains a good collection of earlier literature.

Several works [13-20] discussed both ideal and non-ideal solutions of the PTE problem by adopting different elementary approaches. Chernick [21] obtained ideal solutions of the PTE problem for the cases $k=3,4,5,6$, and 7 including the solutions that Escott [12] had produced for the cases $k=4$ and 5. Chernick also obtained all rational solutions for the case $k=3$ together with the parametric solutions. Burchnall and Chaundy [22] explained how the solutions of the PTE problem could be represented in the form of magic squares. Later, Dorwart and Brown [23] presented some general theorems regarding the existence of solutions of the PTE problem in a more descriptive way. They aimed to give detailed examples instead of proving those theorems and explained how to obtain equivalent solutions once an initial solution was obtained. Dorwart and Brown also proved a beneficial result regarding the least number $\rho$, for which the PTE problem $\left\{u_{1}, u_{2}, \cdots, u_{\rho}\right\}={ }_{k}$ $\left\{v_{1}, v_{2}, \cdots, v_{\rho}\right\}$ possess non-trivial solutions. They provided some examples to ensure $\rho(k)=k+$ 1 for $k=1,2, \cdots, 7$ and tabulated the values for $\rho$ and $k=8,9, \cdots, 24$. Also, they mentioned how the ideal solutions of the PTE problem could be conveniently applied to compute the large number of decimal places occurring in the logarithm of integers. They considered a familiar series $\log _{10} \frac{M}{N}=2 \log _{10} e\left[\frac{M-N}{M+N}+\frac{1}{3}\left(\frac{M-N}{M+N}\right)^{3}+\frac{1}{3}\left(\frac{M-N}{M+N}\right)^{5}+\cdots\right]$. Then they replaced $M$ and $N$ by univariate polynomials with integral roots such that $M-N$ is a constant. They also pointed out a connection between the PTE problem and the derivation of the following formula, used for the computation of $\pi, \tan ^{-1} \frac{a}{x+\alpha}+\tan ^{-1} \frac{b}{x+\beta}+\cdots=\tan ^{-1} \frac{P}{X}$, where $X$ is a real polynomial whose degree and the number of fractions on the left-hand side of the equality are equal. They observed that the problem of reducibility of polynomials of the form $f(x)=a\left(x-x_{1}\right)\left(x-x_{2}\right) \cdots\left(x-x_{n}\right) \pm p$, where $p$ is a prime and $x \neq x_{i}$, is equivalent to finding the ideal solutions of the PTE problem. So, the work in Reference [23] seems to be an important building block in the area of the PTE problem. As a continuation, Dorwart [24] invented some equivalent forms of solutions for ideal solutions with $k=1,2,3$, presented a general non-symmetric solution for $k=3$, and provided a base for finding ideal parametric solutions of the PTE problem with $k \geq 4$.

Another significant contribution in the early stages was from Wright [25]. He provided two elementary proofs for Lehmer's result, while Lehmer's proof depends on the ideas of generating functions, exponential differentiation, matrices, and complex roots of unity. Wright provided two proofs. One depends on the factor theorem for polynomials, and the other depends on the multinomial theorem for a positive integral index. Wright also provided a tool for extracting solutions of the PTE problem with arbitrary degree $k$. He proved that if a non-trivial solution of $\sum_{i=1}^{j} x_{i 1}^{h}=\sum_{i=1}^{j} x_{i 2}^{h}=\cdots=$ $\sum_{i=1}^{j} x_{i s}^{h}$, where $1 \leq h \leq k$ and $k, s \geq 1$, exists for $s=2$ and $i=J$, then we can construct a non-trivial solution for the same $k, s=2^{m}$ and $j=m J$, where $m$ is any positive whole number. He also constructed a solution for general $s$ with $j=m 2^{k}$ provided $s \leq 2^{m}$. Later, Roberts [26] presented a new proof of Lehmer's theorem by using difference operators and provided some solutions of the PTE problem as a particular case. The proof given in Reference [26] was more general than the already existing results.

Additionally, Wright [27] provided a direct proof for Prouhet's generalization. One of the major results in Reference [27] was as follows: "Express each $n\left(0 \leq n \leq j^{k+1}-1\right)$ as a decimal in the scale of $j$. If the least positive residue to modulus $j$ of the sum of the digits of $n$ in this scale is $v$, then assign $n$ to the set $S_{v}$. Then each of the sets $S_{v}$ contains just $j^{k}$ members, which may be taken as $x_{1 v}, x_{2 v}, \cdots$ and which satisfy the simultaneous equations $\sum_{i=1}^{s} x_{i 1}^{k}=\sum_{i=1}^{s} x_{i 2}^{k}=\cdots \sum_{i=1}^{s} x_{i j}^{h}$, with $s=j^{k}$ where $k \geq 1$, $j \geq 2$, and $1 \leq h \leq k . "$ After two years, Melzak [28] implemented a method for estimating the bound $K_{1}=K(n)$, the least integer in the set of all $s$ (size of the problem) that possesses a solution for the PTE problem, which yields to the actual solution. He proved that " $K(n)=\frac{1}{2} \min _{P \in \alpha} S\left[P(x)(1-x)^{n+1}\right]$, where $\alpha$ is the class of all polynomials having integer coefficients, $N$ is a positive integer, and $S[P]=$ $\sum_{i=0}^{N}\left|a_{i}\right|$ for $P=P(x)=\sum_{i=0}^{N} a_{i} x^{i \prime \prime}$. This gives an exact expression for finding an estimate for $K(n)$ 
rather than computing the value of $K(n)$. The proof given in Reference [28] was non-constructive, and also could not be employed to compute $K(n)$, however it led to better-bound estimates of $K(n)$ than those previously existing. He also provided a list of estimates for $K(n)$ for different $n$. Later, Barrodale [29] provided some new solutions to the problem with the help of computers, by improving the lower bounds that had been found by Melzak. He extended the results to $k \leq 85$. He also discussed some extension of the PTE problem by defining $M(k)$ as the least value of $s$ such that $\sum_{i=1}^{s} a_{i}^{j}=\sum_{i=1}^{s} b_{i}^{j}$, where $(j=1,2, \cdots, k)$, has a solution together with $a_{1}^{k+1}+\cdots+a_{s}^{k+1} \neq b_{1}^{k+1}+$ $\cdots+b_{s}^{k+1}$. Sinha [30] obtained a parametric solution of size 10 and degree 8 by solving the system $\left\{A_{1}, A_{2}, A_{3}, A_{4}, A_{5}\right\}={ }_{l}\left\{B_{1}, B_{2}, B_{3}, B_{4}, B_{5}\right\} ; l=1,3,5,7$ where the $A_{i}$ 's and $B_{i}$ 's are integers. Eggleton and Selfridge [31] created a set of consecutive integers such that the integers do not have comparatively big prime factors based on the PTE problem solutions.

Adler and Robert Li [32] implemented the method of construction of magic cubes of some order $N$ from the generalized Morse-Hedlund sequence, which is a particular case of the Prouhet sequence. Also, they clearly explained how solutions of the PTE problem are associated with magic cubes and concluded by proving a nice result regarding the entries in the magic cubes. According to the result in Reference [32], if a magic cube of order $i^{J}$ is considered, then its conjugate sub-cubes having dimension $n$ satisfy the equal sum of the $k$ th power of their inputs, where $k=0,1,2, \cdots, J n-1$. Later, Bremner [33] geometrically developed the problem of equal sums of fifth powers by studying a K3 surface contained in the fourfold. Compared to other approaches, it was a new one in the study of equal sums of like powers. So it opened a new technique that could be shortly applied in the study of the PTE problem. Thus, the contribution from this work is highly important in the study of the PTE problem. Nine years later, Rees and Smyth [34] studied the constant associated with the PTE problem, which was a new approach in the study of the PTE problem at that time. They proved some divisibility results on the constant $K_{n}$ associated with the PTE problem for $n \leq 10$. They also studied the divisibility of $K_{n}$ modulo a prime power.

After a year, Smyth [35] again studied ideal 9th order multigrades and Letac's elliptic curve. He provided a constructive definition of 9th order multigrades as follows: "9th order multigrades are the solutions $\left\{\left(n_{1}, n_{2}, \cdots, n_{10}\right),\left(m_{1}, m_{2}, \cdots, m_{10}\right)\right\}$ in integers of the system of equations $\sum_{i=1}^{10} n_{i}^{j}=$ $\sum_{i=1}^{10} m_{i}^{j}$ for $j=1,2, \cdots, 9^{\prime \prime}$. Later, Borwein and Ingalls [36] presented some new limits for the Easier-Waring problem, irrespective of the existing bounds and also for the PTE problem of small size. They also presented an algorithm for finding ideal symmetric $p$-adic solutions for the PTE problem and with the help of those algorithms, they started to find an ideal symmetric solution of size 11. Jacroux [37] derived some new results on the construction of sets of integers with equal power sums. He explained the constructive method as follows: "Let $S_{0}, S_{1}, \cdots, S_{L-1}$ and $T_{0}, T_{1}, \cdots, T_{L-1}$ be sets of integers with $\left|S_{0}\right|=\left|S_{1}\right|=\cdots=\left|S_{L-1}\right|=w,\left|T_{0}\right|=\cdots=\left|T_{L-1}\right|=z$ such that $S_{0}={ }_{p} S_{1}={ }_{p} \cdots={ }_{p} S_{L-1}$ and $T_{0}={ }_{q} T_{1}={ }_{q} \cdots=_{q} T_{L-1}$. Then $\overline{S_{0}}={ }_{p+q+1} \bar{S}_{1}={ }_{p+q+1} \cdots={ }_{p+q+1} S_{L-1}^{-}$where for $m=$ $0,1 \cdots L-1, \widehat{S_{m}}=\cup_{i=0}^{L-1} \cup_{i \in T_{j}, i+j \equiv m(\bmod L)}\left\{S_{i}+(t-1) L w\right\}$ and $S_{i}+(t-1) L w$ is the set $S_{i}$ with $(t-1) L w$ added to each element". Jacroux also implemented a new idea in partitioning the set of integers, which generalizes Prouhet's following findings: "Let $n_{1}=L d$. Then the first $n_{1}$ positive integers can be partitioned into $L$ classes $S_{0}, \cdots, S_{L-1}$ of size $d$ such that $S_{0}={ }_{1} \cdots={ }_{1} S_{L-1}$ if and only if $L$ is odd or $d$ is even." Ekl [38] employed a computational technique to find the solution of $a_{1}^{7}+a_{2}^{7}+a_{3}^{7}+a_{4}^{7}=b_{1}^{7}+b_{2}^{7}+b_{3}^{7}+b_{4}^{7}$. Maltby [39] studied the relation between pure product polynomials and the PTE problem. In the theory of pure product polynomials, the PTE problem is to find multigrades of the smallest possible size for each degree.

A lot of work in different directions on the PTE problem has been done from 2000 onwards. Choudhry [40] introduced the complete ideal symmetric solutions of the 4th-degree PTE problem. He discussed the non-symmetric solution for $k=4$ and used it to obtain a parametric solution of the corresponding Diophantine system $\sum_{i=1}^{5} a_{i}^{r}=\sum_{i=1}^{5} b_{i}^{r}$ for $r=1,2,3,4$, and 6. Later, Borwein [41] provided a fundamental tool in computational techniques that could be applied to the PTE problem. 
Also, he presented a list of the smallest known ideal solutions of size 2 to 12 , excluding the case of size 11. Figure 1 illustrates these solutions.

$$
\begin{aligned}
{[ \pm 2] } & ={ }_{1}[ \pm 1] \\
{[-2,-1,3] } & ={ }_{2}[2,1,-3] \\
{[-5,-1,2,6] } & ={ }_{3}[-4,-2,4,5] \\
{[-8,-7,1,5,9] } & ={ }_{4}[8,7,-1,-5,-9] \\
{[ \pm 1, \pm 11, \pm 12] } & ={ }_{5}[ \pm 4, \pm 9, \pm 13] \\
{[-50,-38,-13,-7,24,33,51] } & ={ }_{6}[50,38,13,7,-24,-33,-51] \\
{[ \pm 5, \pm 14, \pm 23, \pm 24] } & ={ }_{7}[ \pm 2, \pm 16, \pm 21, \pm 25] \\
{[174,148,132,50,8,-63,-119,-161,-169] } & ={ }_{8}[-174,-148,-132,-50,-8,63,119,161,169] \\
{[ \pm 99, \pm 100, \pm 188, \pm 301, \pm 313] } & ={ }_{9}[ \pm 71, \pm 131, \pm 180, \pm 307, \pm 308] \\
{[ \pm 103, \pm 189, \pm 366, \pm 452, \pm 515] } & ={ }_{9}[ \pm 18, \pm 245, \pm 331, \pm 471, \pm 508] \\
{[ \pm 151, \pm 140, \pm 127, \pm 86, \pm 61, \pm 22] } & ={ }_{11}[ \pm 148, \pm 146, \pm 121, \pm 94, \pm 47, \pm 35] \\
&
\end{aligned}
$$

Figure 1. List of the smallest known ideal solutions of size upto 12, as in Reference [41].

Later, Borwein et al. [42] explained a computational technique for finding the ideal symmetric solutions to the PTE problem. They considered Theorem 6 in order to reduce the search limit. They started with Newton's iteration and a value above the search limit, and continued until either the iteration converges, the iteration fails to monotonically decrease, or the 60th iteration was reached. They obtained solutions of sizes up to 12 and presented two new solutions of size 10.

Choudhry [43] extended previous analysis and developed ideal non-symmetric solutions of the PTE problem of degrees four and five. He also discussed the parametric solutions of the Diophantine system related to these problems. He obtained a much simpler parametric solution of degree four than in Reference [40]. Again, Choudhry [44] studied the solutions of the Diophantine system connected to the PTE problem with no non-trivial solutions. Later, Choudhry [45] introduced the matrix analogs of the PTE problem by considering the problem over $2 \times 2$ matrices over $\mathbb{Z}, M_{2}(\mathbb{Z})$. Srikanth and Gopalan [46] obtained a general form of non-ideal, non-trivial, parametric integral solutions of the PTE problem with degree $k=2$ and size $n=4$.

In 2007, Alpers and Tijdeman [47] studied the two-dimensional Tarry-Escott problem. They presented parametric solutions for the cases $n=2,3,4,6$ and proved the Prouhet theorem for the two-dimensional case. Also, they proved that the solutions of the two-dimensional problem give rise to solutions of the classical PTE problem. They discussed the geometrical aspects of the PTE problem by obtaining solutions from convex $2 n$-gons whose vertices belong to $\mathbb{Z}^{2}$ and also introduced the study of the classical PTE problem over Gaussian integers.

Choudhry and Wróblewski [48] introduced an idea to obtain solutions to the PTE problem of degree 11, from the solutions of the system $\sum_{i=1}^{k} A_{i}^{k}=\sum_{i=1}^{k} B_{i}^{k}$ for $k=2,4,6,8,10$. Also, they proved new results on the sum of thirteenth powers with the help of those ideal solutions. At the end of their research article, they showed that any integer could be written as an addition or subtraction of 13th powers of at most 27 positive integers. Prugsapitak [49] studied the PTE problem over a quadratic field. Again, Choudhry [50] produced the complete ideal symmetric solution of the 4th-degree PTE problem and proved that any integer $n$ could be represented as $n=x_{1}+x_{2}+x_{3}+x_{4}$ where $x_{1}, x_{2}, x_{3}, x_{4}$ are distinct non-zero integers satisfying $x_{1}^{3}+x_{2}^{3}+x_{3}^{3}+x_{4}^{3}=n^{3}$. 
Two years later, Prugsapitak [51] determined the complete ideal solutions of the PTE problem of degree two over both $\mathbb{Z}[i]$, the ring of Gaussian integers, and $F_{p}[x]$, the ring of polynomials over a finite field $F_{p}$ where $p$ is a prime. A different approach to that of Cayley [52,53] was adopted. Also, a discussion on obtaining ideal solutions over $\mathbb{Z}[i]$ from the integer ideal solutions of degree two and from the proper integer solutions of the two-dimensional PTE problem was performed. The developed method could be used to get infinitely many solutions of degree two over $\mathbb{Z}[i]$. Cayley $[52,53]$ implemented solutions of the classical PTE problem over Gaussian integers. He performed both the theoretical and computational techniques to analyze the ideal solutions over $\mathbb{Z}[i]$ and provided some divisibility results on the constant associated with the PTE problem over $\mathbb{Z}[i]$ and $\mathbb{Z}$. Table 1 provides the list of primes dividing the constant $K_{n}$ associated with the ideal symmetric solutions of the classical PTE problem.

Table 1. Divisibility of $K_{n}$.

\begin{tabular}{cc}
\hline $\boldsymbol{n}$ & Some Primes Dividing $K_{\boldsymbol{n}}$ \\
\hline 9 & $2,3, \cdots, 13$ \\
10 & $2,3,5,7,13,17,23$ \\
11 & $2, \cdots, 19,31$ \\
12 & $2, \cdots, 11,17,19,29$ \\
13 & $2, \cdots, 41$ \\
\hline
\end{tabular}

Černý [54] presented a class of solutions to the PTE problem. Prouhet's solution was described as a special case of that class. Again, Choudhry [55] provided a detailed study of the PTE problem by considering a new condition $u_{1} u_{2} \cdots u_{s}=v_{1} v_{2} \cdots v_{s}$ together with the already existing conditions of the PTE problem. He provided numerical solutions for many Diophantine systems of this type. Additionally, he showed that certain Diophantine systems with equal sums of powers and equal products could not have any non-trivial solutions.

Later, Kongsiriwong and Prugsapitak [56] investigated the number of ideal solutions to the second-degree PTE problem over the finite field $\mathbb{Z}_{p}$, where $p$ is a prime. They presented the non-trivial integer solutions of the corresponding Diophantine system $\sum_{j=1}^{3} x_{j}^{k}=\sum_{j=1}^{3} y_{j}^{k}$, for $k=1,2,5$. They first established the result over $\mathbb{Z}_{p}$, where $p>5$ is an odd prime, using Newton's identities that relate the two types of symmetric polynomials. Choudhry [57] extended his previous studies to obtain the minimum number of terms required for the Diophantine system $\sum_{j=1}^{r_{1}} x_{j}^{l}=\sum_{j=1}^{r_{2}} y_{j}^{l}$, where $l=$ $1,2, \cdots, k$, associated with the PTE problem $\sum_{j=1}^{r} x_{j}^{l}=\sum_{j=1}^{r} y_{j}^{l}$, where $l=1,2, \cdots, k$.

Nguyen [58] showed that the solution of the PTE problem can be obtained by deriving the generalization of the product generating function for the Prouhet-Thue-Morse sequence. Also, he presented some applications of Prouhet's solutions in the radar field and he provided an elementary and straightforward proof for results regarding the generalization of the product generating function. Černý [59] again proved that the structure of a multi-dimensional word obtained by a composition of a finite sequence of morphism-like mappings, out of which $k$ were balanced, yields a solution to the multi-dimensional PTE problem of degree $k$. Also, he revealed the solutions to the multi-dimensional PTE problem over an arbitrary semi-ring.

The application of the gravitational search algorithm (GSA) to find numerous solutions of the PTE problem was investigated by Venkatesan et al. [60]. They provided computational results to establish that heuristic methods like GSA give high-quality solutions to the PTE problem. The bridge between non-polynomial (NP)-hardness of the Reed-Solomon decoding and the PTE problem was the subject of Gandikota et al. [61]. They also discussed the existence of inhomogeneous PTE solutions over an arbitrary field. Laughlin and Zimmer [62] provided a relation between the well poised (WP)-Bailey pair and the PTE problem. They also produced a simple example to illustrate the relationship. They used the generalized telescoping approach to prove their results on the PTE problem. Also, they noted that the ideal solutions of the PTE problem yield an infinite summation formula, with a suitable example. 
Recently, Filaseta and Markovich [63] studied the 2-adic values of the constant associated with the PTE problem. They produced the 2-adic values for the cases $n=8$ and $n=9$ using the classical theory of the Newton polygon. For the case $n=8$, they found a lower bound; whereas for the case $n=9$, they succeeded to produce the exact value. Again, Choudhry [64] found out some new parametric ideal solutions of the problem with degree less than or equal to 7, by applying the concept of arithmetic progressions. He invented some new parametric solutions of the Diophantine systems associated with the PTE problem, which he considered as a specimen for the research. Also, he suggested that the method can be extended to the cases when $k=4,5$ and $k \geq 8$, and he left it as an open problem. Shuwen [65] provided a list of ideal symmetric solutions of the PTE problem for $k=1,2, \cdots, 9,11$ and ideal non-symmetric solutions for $k=2,3, \cdots, 7$ together with some elementary theorems.

Thus, a detailed survey has been performed related to the PTE problem which summarizes the research space. All of this work collectively provides a good picture of the development of significant outcomes in this area.

\section{Applications}

The ideal solutions of the PTE problem play a major role in fields like combinatorics, computer science, the easier waring problems, andin finding the rational points on elliptic curves. There are some applications of solutions of the PTE problem on trace reconstruction problems. Krasikov and Roditty [66] established the k-reconstructibility of words of length $n$ for $k \geq\left\lfloor\frac{16}{7} \sqrt{n}\right\rfloor+5$ by dealing with words only over the alphabet $\{0,1\}$. They also proved the necessary condition for non-reconstructibility of words of length $n$. Later, Borwein et al. [67] proved that the classical PTE problem could be rephrased as the problem of finding the maximal vanishing at 1 of a polynomial with integer coefficients and with $l_{1}$ norm $2 n$. Later, Foster and Krasikov [68] dealt with the problem of finding, given $n$, the smallest $m$ such that there exists a polynomial $\phi(x)$ of degree $m$ satisfying $|\phi(-1)|>\sum_{i=0}^{n}|\phi(i)|$ and showed that $m \geq\lfloor\sqrt{n \ln 2}-1 \mid$. Also, they improved the value of $m$ for polynomials non-negative on $[0, n]$, compared to the result in Reference [67], and they sharpened the bounds concerning the PTE problem and the sequence reconstruction problem.

Another important application of the PTE problem is its relation with tomography. The first published result on the relation between a particular PTE problem and tomography can be seen in Alpers' Ph.D. thesis [69]. Also, he showed that the switching components yield PTE solutions for specific values of $(k, n)$. Hernández and Luca [70] showed how graph theory relates to the ideal solutions of the PTE problem. They proved the necessary and sufficient condition for the existence of integer roots of chromatic polynomials of a non-chordal graph with ideal solutions of the PTE problem. Also they proved that, if a family $\mathbb{L}$ of graphs whose chromatic polynomials have integer roots is considered, then $G_{\alpha_{1}, \alpha_{2}, \cdots, \alpha_{k}} \in \mathbb{L}$ if and only if the degree of the ideal solution of the PTE problem is $k-1$, for $k \geq 3$. Here, $G_{\alpha_{1}, \alpha_{2}, \cdots, \alpha_{k}}$ stands for the graph made from the disjoint union of $k$ complete graphs on $\alpha_{1}, \alpha_{2}, \cdots, \alpha_{k}$ vertices. Later, Broadhurst [71] found ten consecutive factorizations by using the solutions of the ideal PTE problem. He also explained the implementation of the Chinese remainder theorem in the study of the ideal PTE problem by producing some examples, and obtained a new solution to degree 12, with the help of computer programs.

Another interesting application of the PTE problem was provided by Alpers and Tijderman [47]. They established a direct connection between tomography and a generalization of the PTE problem. They introduced a more general $\mathrm{PTE}_{d}$ problem as follows: "Given natural numbers $k, n, d$, find two different multi sets $X:=\left\{\xi_{1}, \cdots, \xi_{n}\right\}, Y:=\left\{\eta_{1}, \cdots, \eta_{n}\right\}$ both contained in $\mathbb{Z}^{d}$ and $\xi_{l}=\left(\xi_{l 1}, \cdots, \xi_{l d}\right)^{T}$, $\eta_{l}=\left(\eta_{l 1}, \cdots, \eta_{l d}\right)^{T}$ for $l \in[n]$ such that $\sum_{l=1}^{n} \xi_{l 1}^{j 1} \xi_{l 2}^{j 2} \cdots \xi_{l d}^{j d}=\sum_{l=1}^{n} \eta_{l 1}^{j 1} \eta_{l 2}^{j 2} \cdots \eta_{l d}^{j d}$, for all non-negative integers $j_{1}, \cdots, j_{d}$ with $j_{1}+j_{2}+\cdots+j_{d} \leq k$." Another major work was from McLaughlin [72]. He showed how an amazing identity of Ramanujan was related to the ideal solutions of the PTE problem of size six. He proved that if a sequence of integers $a_{k}, b_{k}, c_{k}, d_{k}, e_{k}, f_{k}, p_{k}, q_{k}, r_{k}, s_{k}$, 
and $t_{k}$ are defined by some generating functions say, $\frac{x^{2}-164 x+3}{x^{3}-99 x^{2}+99 x-1}=\sum_{k=0}^{\infty} a_{k} x^{k}$, $\frac{-7 x^{2}+134 x+1}{x^{3}-99 x^{2}+99 x-1}=\sum_{k=0}^{\infty} b_{k} x^{k}, \frac{-x^{2}+298 x-1}{x^{3}-99 x^{2}+99 x-1}=\sum_{k=0}^{\infty} c_{k} x^{k}, \frac{-5 x^{2}+228 x-7}{x^{3}-99 x^{2}+99 x-1}=$ $\sum_{k=0}^{\infty} d_{k} x^{k}, \quad \frac{3 x^{2}+258 x-5}{x^{3}-99 x^{2}+99 x-1}=\sum_{k=0}^{\infty} e_{k} x^{k}, \quad \frac{-3 x^{2}+94 x-3}{x^{3}-99 x^{2}+99 x-1}=\sum_{k=0}^{\infty} f_{k} x^{k}$, $\frac{-5 x^{2}+138 x+3}{x^{3}-99 x^{2}+99 x-1}=\sum_{k=0}^{\infty} p_{k} x^{k}, \frac{3 x^{2}+244 x+1}{x^{3}-99 x^{2}+99 x-1}=\sum_{k=0}^{\infty} q_{k} x^{k}, \frac{x^{2}+254 x-7}{x^{3}-99 x^{2}+99 x-1}=$ $\sum_{k=0}^{\infty} r_{k} x^{k}, \frac{-7 x^{2}+148 x-5}{x^{3}-99 x^{2}+99 x-1}=\sum_{k=0}^{\infty} s_{k} x^{k}$, and $\frac{3}{1-x}=\sum_{k=0}^{\infty} t_{k} x^{k}$, then for $1 \leq j \leq 5$ and each $k \geq 0, a_{k}^{j}+b_{k}^{j}+c_{k}^{j}+d_{k}^{j}+e_{k}^{j}+f_{k}^{j}-p_{k}^{j}-q_{k}^{j}-r_{k}^{j}-s_{k}^{j}-t_{k}^{j}=1$. From the example given in Reference [72], one could easily recognize the relation between the Ramanujan identity and the PTE problem.

A nice application of the PTE problem in the field of computer science was clearly depicted by Borchert et al. [73]. They studied complete factorization of polynomials of degree 4, 8, and 16 into distinct roots with the help of electronic circuits. Also they proved how one could generate new solutions for the PTE problem using circuits and product gates. Alpers and Larman [74] derived some bounds on the sizes of solutions to both the classical PTE problem and the two-dimensional PTE problem. They also reported that polynomial size solutions for the two-dimensional PTE problem exist. The result developed by them, that sharpens the bound on the size of solutions is as follows: "For every $\epsilon>0$, there exists a constant $c>0$ such that there are solutions of the two-dimensional PTE problem of degree $k$ and size bounded by $c k^{3+\epsilon "}$. Additionally, they remarked at the end of their article that solutions of the classical PTE problem could be obtained by applying a suitable linear functional to two-dimensional PTE solutions that maps $\left(\xi_{1}, \xi_{2}\right) \in \mathbb{Z}^{2}$ to $\alpha_{1} \xi_{1}+\alpha_{2} \xi_{2}$, where $\alpha_{1}, \alpha_{2} \in \mathbb{Z}$ are suitably chosen.

One of the applications of PTE solutions in combinatorics is the formation of the generalized Thue-Morse sequence to finite alphabets using PTE partitions of arbitrarily high symmetry. Bolker et al. [75] showed how such partitions could be used to pour the same volume of coffee from a container into a finite number of cups so that each gets almost the same amount of caffeine. The basic idea used in Reference [75] was as follows: "To fill two cups with four pours use the word ABBA: pour the first and last quarters into cup $A$ and the second and third quarters into B. For eight pours the magic word is ABBA BAAB. Continue recursively by appending to each sequence of length $n$ its complement (in the obvious sense) in order to find the optimal partition for pourings using $2^{k}$ subdivisions. Collecting all the solutions into an infinite word AB BA BAAB BAABABBA $\cdots$ produces the Thue-Morse sequence. Richman's argument, showing that the word ABBA BAAB solves the two cup problem using eights, depends essentially on $1+4+6+7=2+3+5+8$ and $1^{2}+4^{2}+6^{2}+7^{2}=2^{2}+3^{2}+5^{2}+8^{2}$. These equations say that the partition $\{\{1,4,6,7\},\{2,3,5,8\}\}$, whose blocks are the positions of A and B in the magic word, solves an instance of the PTE problem." With this idea, Bolker et al. generalized the recursive construction of the Thue-Morse sequence in order to generate a new family of solutions to the PTE problem.

Recently, Alpers [76] discussed the PTE problem in a 2018 habilitation thesis, which contains another recent review of the PTE problem. It included the different tomographic applications in aspects of Diophantine number theory, tomographic super-resolution imaging, tomographic point tracking, tomographic reconstructions of polycrystals, and geometric methods for electron tomography.

\section{Open Problems}

In this section, the authors address some open problems found during the literature survey. An important open problem in the PTE problem is a conjecture of Wright [17] that for every natural number $n \geq 3$, an ideal solution exists.

- Does an ideal solution exist for the PTE problem of size beyond 13? Is it possible to extend the algorithm found by Cayley [52,53] to find the solutions of larger size?

The algorithm used in Reference [52] was an extension of the algorithm for finding odd and even 
symmetric solutions to the PTE problem over $\mathbb{Z}$, which was implemented in Reference [42]. Cayley used Maple and $\mathrm{C}++$ software to develop the algorithm. The computer search was implemented to find solutions with real and imaginary parts between 0 and 30 for the sizes 10 and 12. Cayley also suggested implementing the algorithm over any ring of integers of a quadratic number field.

- Solve for ideal solutions by considering modulo $p^{n}$ for all primes not equal to the solution size. By considering modulo $p$, the search space can be reduced to a convenient size for higher degrees. So, finding modulo $p^{n}$ of ideal solutions is appreciated.

- Wright [17] showed that there are solutions of quadratic size by a non- constructive method for every degree. Are there constructive methods? Is it possible to lower the bound to something smaller than quadratic?

Wright proved that $N(k) \leq \frac{1}{2}\left(k^{2}+3\right)$ if $k$ is odd and $N(k)=\frac{1}{2}\left(k^{2}+4\right)$ if $k$ is even, where $N(k)$ is the least value of $j$ such that the equations $a_{1}^{h}+\cdots+a_{j}^{h}=b_{1}^{h}+\cdots+b_{j}^{h}$, for $1 \leq h \leq k$, have a non-trivial solution.

- Does there exists a 2-adic ideal solution for every $n \geq 3$ ? Does a $p$-adic ideal solution necessarily exist for $n=11$, where $p$ is a prime?

Filaseta and Markovich [63] found the 2-adic solutions of the PTE problem using the classical theory of Newton polygons. If $\overline{C_{n}}$ denotes the greatest common divisor (GCD). over all constants $K_{n}$ associated with the PTE problem and $v_{2}\left(\bar{C}_{n}\right)$ denotes the 2-adic value of $\overline{C_{n}}$, they proved that $v_{2}\left(\bar{C}_{8}\right) \geq 6$ and $v_{2}\left(\bar{C}_{9}\right)=9$.

- Is it possible to generate a true algorithm that determines whether there is a solution of size 11? Presently, there is no algorithm for finding a solution for size 11. If one can generate such solutions then it will be a great contribution to the PTE problem.

- Is it possible to find solutions to PTE problem that follow some specific patterns similar to the findings of Choudhry [64]?

Choudhry found the solutions of the PTE problem that follow arithmetic progression (AP). He established the solutions for $k \leq 7$ using the software Maple.

- If we define $N(k)$ as the least $n$ such that the PTE problem of degree $k$ has a solution of size $n$, then is it possible to find a lower bound on $N(k)$ that would rule out ideal solutions?

There were a lot of studies $[17,28,36]$ on the upper bounds for $N(k)$. If one can find a lower bound to this problem, then it will be a great contribution.

\section{Conclusions}

The classical PTE problem is a vast research area in number theory. Almost all the available literature on the PTE problem has been reviewed. Different approaches to find the solutions to the PTE problem, both in the ideal and non-ideal cases, have been observed. All the ideal symmetric, ideal non-symmetric, and ideal prime solutions for different $k$ values are tabulated to the best of the authors' knowledge. It is found that there exists a generic way of finding a PTE solution of degree $n$, and size $2^{n}$. Also, some applications, as well as open problems existing in the study of the PTE problem, are addressed. Even though there are solutions to the PTE problem up to size 12, no solutions of size 11 and size greater than 12 are reported to the best of the authors' knowledge. Thus, there are still a lot of aspects that need to be studied related to the PTE problem.

Author Contributions: Conceptualization, investigation, and paper writing V.N.; Editing and over all supervision S.R. Funding: This research received no external funding.

Acknowledgments: The authors gratefully acknowledge TATA Realty-SASTRA Srinivasa Ramanujan Research Chair for supporting this research work.

Conflicts of Interest: The authors declare no conflict of interest. 


\section{Abbreviations}

The following abbreviations are used in this manuscript:

PTE Prouhet Tarry Escott Problem

NP Non-Polynomial

WP Well Poised

GCD Greatest Common Divisor

AP Arithmetic Progression

GSA Gravitational Search Algorithm.

\section{References}

1. Srikanth, R.; Subburam, S. Super elliptic equations of the form $y^{p}=x^{k} p+\cdots+a_{0}$. J. Algebra Number Theory Acad. 2012, 2, 331-335.

2. Srikanth, R.; Subburam, S. The solution free diophantine equation $y^{2}=a_{n} x^{n}+\cdots+a_{0}$. Int. J. Math. Anal. 2012, 6, 2591-2596.

3. Srikanth, R.; Subburam, S. On the diophantine equation $y^{p}=f\left(x_{1}, x_{2}, \cdots, x_{r}\right)$. Funct. Approx. Comment. Math. 2018, 58, 37-42. [CrossRef]

4. Frolov, M. Égalités à deux degrés. Bull. Soc. Math. Franc. 1889, 17, 69-83. [CrossRef]

5. Escott, E.B. The calculation of logarithms. Quart. J. Math. 1910, 41, 147-167.

6. Tarry, G. Questions 4100. Intermed. Math. 1912, 19, 200.

7. Bastien, L. Impossibilité de $u+v \stackrel{3}{=} x+y+z$. Sphin X-Oedipe 1913, 8,171-172.

8. Gloden, A. Mehrgradige Gleichungen, 2nd ed.; P. Noordhoff: Groningen, The Netherlands, 1943.

9. Allouche, J.-P.; Shallit, J. The Ubiquitous Prouhet-Thue-Morse sequence. In Sequences and Their Applications; Springer: London, UK, 1999; pp. 1-16.

10. Euler, L. Letter to Goldbach, Sep 4, 1751. Corresp. Math. Phys. (ed. Fuss). 1843, 1, 549-552. Available online: http:/ / eulerarchive.maa.org/correspondence/ (accessed on 25 November 2018).

11. Goldbach, C. Letter to Euler, July 18, 1750. Corresp. Math. Phys. (ed. Fuss). 1843, 1, 525-526. Available online: http: / / eulerarchive.maa.org/ correspondence/ (accessed on 25 November 2018).

12. Dickson, L.E. Sets of integers with equal sums of like powers. In History of the Theory of Numbers; Chelsea: New York, NY, USA, 1971; Volume 2, Chapter 24, pp. 705-716.

13. Lehmer, D.H. The Tarry-Escott problem. Scr. Math. 1947 13, 37-41.

14. Sinha, T.N. A note on theorem of Lehmer. J. Lond. Math. Soc. 1972, s2-4, 541-544. [CrossRef]

15. Wright, E.M. Equal sums of like powers. Bull. Am. Math. Soc. 1948, 54, 755-757. [CrossRef]

16. Kleiman, H. A Note on the Tarry-Escott problem. J. Reine. Angew. Math. 1975, 278-279, 48-51.

17. Wright, E.M. On Tarry's Problem (I). Quart. J. Math. 1935, 6, 261-267. [CrossRef]

18. Wright, E.M. On Tarry's Problem (III). Quart. J. Math. 1937, 8, 48-50. [CrossRef]

19. Mirsky, L. A remark on D.H.Lehmers solution of the Tarry-Escott problem. Scr. Math. 1948, 14, $126-127$.

20. Palamá, G. On a theorem of D.H.Lehmer concerning the Tarry-Escott Problem. Scr. Math. 1953, 19, 19-23.

21. Chernick, J. Ideal solutions of the Tarry- Escott Problem. Am. Math. Mon. 1937, 44, 626-633. [CrossRef]

22. Burchnall, J.L.; Chaundy, T.W. A type of magic square in Tarry's problem. Quart. J. Math. Oxf. Ser. 1937, 8, 119-130. [CrossRef]

23. Dorwart, H.L.; Brown, O.E. The Tarry- Escott Problem. Am. Math. Mon. 1937, 44, 613-626. [CrossRef]

24. Dorwart, H.L. Sequence of ideal solutions to the Tarry-Escott Problem. Bull. Am. Math. Soc. 1947, 53, $381-391$. [CrossRef]

25. Wright, E.M. Equal Sums of Like Powers. Proc. Edinb. Math. Soc. 1949, 2, 138-142. [CrossRef]

26. Roberts, J.B. A New Proof of theorem of Lehmer. Can. J. Math. 1958, 10, 191-194. [CrossRef]

27. Wright, E.M. Prouhet's 1851 solution of the Tarry-Escott problem of 1910. Am. Math. Mon. 1959, 102, $199-210$. [CrossRef]

28. Melzak, Z.A. A Note on the Tarry-Escott Problem. Can. Math. Bull. 1961, 4, 233-237. [CrossRef]

29. Barrodale, I. The Tarry-Escott Problem. Master's Thesis, University of British Columbia, Vancouver, BC, Canada, 1965.

30. Sinha, T.N. On the Tarry-Escott Problem. Am. Math. Mon. 1966, 73, 280-285. [CrossRef] 
31. Eggleton, R.B.; Selfridge, J.L. Consecutive integers with no large prime factors. J. Aust. Math. Soc. 1976, 22, 1-11. [CrossRef]

32. Adler, A.; Li, S.Y.R. Magic cubes and prouhet sequences. Am. Math. Mon. 1977, 84, 618-627. [CrossRef]

33. Bremner, A. A geometric approach to equal sums of fifth powers. J. Number Theory 1981, 13, 337-354. [CrossRef]

34. Rees, E.; Smyth, C. On the constant in the Tarry-Escott problem. In Lecture Notes in Mathematics 1415; Springer: Berlin, Germany, 1990; pp. 196-208 .

35. Smyth, C.J. Ideal 9th-order multigrades and Letac's elliptic curve. Math. Comp. 1991, 57, 817-823.

36. Borwein, P.; Ingalls, C. The Prouhet-Tarry-Escott Problem revisited. Enseign. Math. 1994, 40, 3-27.

37. Jacroux, M. On the construction of sets of integers with equal power sums. J. Number Theory 1995, 52, 35-42. [CrossRef]

38. Ekl, R.L. Equal Sums of four seventh powers. Math. Comp. 1996, 65, 1755-1756. [CrossRef]

39. Maltby, R. Pure product polynomials and the Prouhet-Tarry-Escott Problem. Math. Comp. 1997 66, 1323-1340. [CrossRef]

40. Choudhry, A. Ideal solutions of the Tarry-Escott problem of degree four and a related Diophantine System. Enseign. Math. 2000, 46, 313-323.

41. Borwein, P. Computational Excursions in Analysis and Number Theory; CMS Books in Mathematics/Ouvrages de Mathematiques de la SMC: 10; Springer: NewYork, NY, USA, 2002.

42. Borwein, P.; Lisoněk, P.; Percival, C. Computational investigations on the Tarry Escott problem. Math. Comp. 2002, 72, 2063-2070. [CrossRef]

43. Choudhry, A. Ideal solutions of the Tarry-Escott problem of degrees four and five and related Diophantine systems. Enseign. Math. 2003, 49, 101-108.

44. Choudhry, A. A Diophantine System related to the Tarry-Escott Problem with no non-trivial solutions. Indian J. Pure Appl. Math. 2004 35, 1395-1398.

45. Choudhry, A. Matrix analogues of the Tarry-Escott problem, multigrade chains and the equation of Fermat. Math. Stud. 2006, 75, 215-224.

46. Gopalan, M.A.; Srikanth, R. A Remark on second degree Prouhet-Tarry-Escott problem. Antarctica J. Math. 2006, 3, 217-225.

47. Alpers, A.; Tijdeman, R. The two-dimensional Prouhet-Tarry-Escott problem. J. Number Theory 2007, 123, 403-412. [CrossRef]

48. Choudhry, A. Wróblewski, J. Ideal solutions of the Tarry-Escott Problem of degree eleven with applications to sums of thirteenth powers. Hardy-Ramanujan J. 2008, 31, 1-13.

49. Prugsapitak, S. The Tarry-Escott Problem over Quadratic Fields. Ph.D. Thesis, Department of Mathematics, University of Illinois at Urbana-Champaign, Champaign, IL, USA, 2009.

50. Choudhry, A. Some Diophantine problems concerning Equal Sums of Integers and their Cubes. Hardy-Ramanujan J. 2010, 33, 59-70.

51. Prugsapitak, S. The Tarry-Escott Problem of degree Two. Period. Math. Hung. 2012, 65, 157-165. [CrossRef]

52. Caley, T. The Prouhet-Tarry-Escott problem for gaussian integers. Math. Comp. 2013, 82, 1121-1137. [CrossRef]

53. Caley, T. The Prouhet-Tarry-Escott Problem. Ph.D. Thesis, Deaprtment of Mathematics, University of Waterloo, Waterloo, ON, Canada, 2012.

54. Černý, A. On Prouhet's Solution to the equal powers problem. Theor. Comput. Sci. 2013, 491, $33-46$.

55. Choudhry, A. Equal sums of Like Powers and equal product of integers. Rocky Mt. J. Math. 2013, 43, 763-792. [CrossRef]

56. Kongsiriwong, S.; Prugsapitak, S. On the number of solutions of the Tarry-Escott problem of degree two and the related problem over some finite fields. Period. Math. Hung. 2014, 69, 190-198. [CrossRef]

57. Choudhry, A. Equal Sums of Like Powers with Minimum Number of Terms. arXiv 2016, arXiv:1602.08698.

58. Nguyen, H.D. A new proof of the Prouhet- Tarry-Escott problem. J. Integers 2016, 16, 1-9.

59. Černý, A. Solutions to the multi-dimensional Prouhet-Tarry-Escott Problem resulting from composition of balanced morphisms. Inf. Comp. 2017, 253, 424-435.

60. Venkatesan, D.; Balachandar, S.R.; Srikanth, R.; Kannan, K.; Venkatesh, S.G. Meta-Heuristic method for Solving Tarry-Escott Problem. Int. J. Pharm. Technol. 2016, 8, 22384-22394. 
61. Gandikota, V.; Ghazi, B.; Grigorescu, E. NP-Hardness of Reed-Solomon decoding and the Prouhet-Tarry-Escott Problem. In Proceedings of the IEEE 57th Annaual Symposium on Foundations of Computer Science (FOCS), New Brunswick, NJ, USA, 9-11 October 2016; Volume 1, pp. 760-769.

62. Laughlin, J.M.C.; Zimmer, P. Some applications of a Bailey-Type Transformation. Int. Math. Forum 2010, 5 , 3007-3022.

63. Filaseta, M.; Markovich, M. Newton polygons and the Prouhet-Tarry- Escott Problem. J. Number Theory 2017, 174, 384-400. [CrossRef]

64. Choudhry, A. A New Approach to the Tarry- Escott Problem. Int. J. Number Theory 2017, $13,393-417$. [CrossRef]

65. Shuwen, C. The Prouhet-Tarry-Escott Problem. 2017. Available online: http://eslpower.org/TarryPrb.htm (accessed on 20 October 2018).

66. Krasikov, I.; Roditty, Y. On a reconstruction for sequences. J. Comb. Theory 1997, 77, 344-348. [CrossRef]

67. Borwein, P.; Erdéyl, T.; Kós, G. Littlewood type problems on [0,1]. Proc. Lond. Math. Soc. 1999, 79, $22-46$. [CrossRef]

68. Foster, W.; Krasikov, I. An improvement of a Borwein-Erdéyl-Kós result. Methods Appl. Anal. 2000, 7, 605-614.

69. Alpers, A. Instability and Stability in Discrete tomography (Published by Shaker Verlag, ISBN 3-8322-2355-X). Ph.D. Thesis, Zentrum Mathematik:Technische Universität München, München, Germany, 2003.

70. Hernández, S.; Luca, F. Integer Roots of chromatic polynomials of non-chordal graphs and the Prouhet-Tarry-Escott Problem. Graphs Comb. 2005, 21, 319-323. [CrossRef]

71. Broadhurst, D. A Chinese Prouhet-Tarry-Escott Solution. 2007. Available online: http://physics.open.ac.uk/ $\sim$ dbroadhu/cpte.pdf (accessed on 20 October 2018).

72. McLaughlin, J. An Identity motivated by an amazing identity of Ramanujan. Fibonacci Q. 2010, 48, 34-38.

73. Borchert, B.; McKenzie, P.; Reinhardt, K. Few Product gates but many zeros. Chic. J. Theor. Comput. Sci. 2013, 2, 1-22.

74. Alpers, A.; Larman, D.G. The Smallest sets of points not determined by their X-rays. Bull. Lond. Math. Soc. 2015, 47, 171-176. [CrossRef]

75. Bolker, E.D.; Offner, C.; Richman, R.; Zara, C. The Prouhet-Tarry-Escott Problem and generalized Thue-Morse sequences. J. Comb. 2016, 7, 117-133. [CrossRef]

76. Alpers, A. On the Tomography of Discrete Structures: Mathematics, Complexity, Algorithms and Its Applications in Materials Science and Plasma Physics. Cummulative Habilitation Thesis, 2018. Available online: https:/ / mediatum.ub.tum.de/1441933 (accessed on 20 January 2019). 